\title{
Nanostructural Characterization of CdTe Thin Film Photovoltaics using Electron Backscatter Diffraction
}

\author{
M.M. Nowell,* J.O. Carpenter,* and S.I. Wright* \\ * EDAX-TSL 392 East 12300 South Suite H Draper UT 84020 USA
}

Thin film photovoltaic (PV) devices, which convert solar radiation into useable electric current, are considered commercially and technologically viable sources of alternative energy. Because these devices are fabricated as thin films, they use less active material than traditional bulk devices and can also be deposited on lower cost substrates. Both of these factors help reduce the overall cost per kilowatt hour [1]. Cadmium Telluride (CdTe) is one thin film PV material that has high optical absorption, a near-ideal band gap, and has been successfully commercialized. CdTe devices also exhibit an interesting microstructural behavior: polycrystalline films have higher conversion efficiencies than single crystal devices [2]. This behavior is also observed in chalcopyrite-structured cells like CIGS.

Grain boundaries in CdTe devices are thought to act as accumulation sites for defects and impurities. This can lead to better quality crystalline lattice within the interior of the grains and the development of a localized electrical field around grain boundaries which can improve the separation and collection of electron hole pairs generated by the photovoltaic process. This leads to enhanced current flow at grain boundaries which has been observed by a variety of analytical technique [3-4]. However not all grain boundaries exhibit the same electrical properties [5-6]. Better understanding of the grain boundary structure is necessary to correlate structure-performance behavior.

Orientation Imaging Microscopy (OIM) uses the automated collection and analysis of Electron Backscattered Diffraction (EBSD) patterns to measure the crystallographic orientation on a nanometer scale when combined with a modern Field Emission Scanning Electron Microscope (FESEM). Orientation information from adjacent grains can be used to derive grain boundary information and when collected from a large number of grains the grain boundary character distribution can be accurately described.

CdTe films are typically treated with a $\mathrm{CdCl}_{2}$ solution and annealed in order to induce grain growth and activate the grain boundaries. In this study, CdTe films were examined with OIM in the asdeposited, partially treated, and fully treated states in order to understand the microstructural and grain boundary character evolution that occurs. Nanometer scale twins were observed in both the asdeposited and partially treated films, and larger scale twins observed in all samples. The practical aspects of this nanoscale characterization using EBSD will be discussed. The effect of twinning on effective grain size was examined as well.

References

[1] J. Poortmans and V. Arkhipov, Thin Film Solar Cells: Fabrication, Characterization, and Applications, John Wiley \& Sons Ltd., West Sussex, 2006.

[2] Visoly-Fisher, I., et al., Advanced Functional Materials, 16 (2006) 649.

[3] Visoly-Fisher, I., et al., Applied Physics Letters, 82 (2003) 556.

[4] Baier, N., et al., Nuclear Instruments and Methods in Physics Research A, 576 (2007) 5. 
[5] Abou-Ras, D., et al., Thin Solid Films, 517 (2009) 2545.

[6] Marron, D.F., et al., Physical Review B, 71 (2005) 033306.

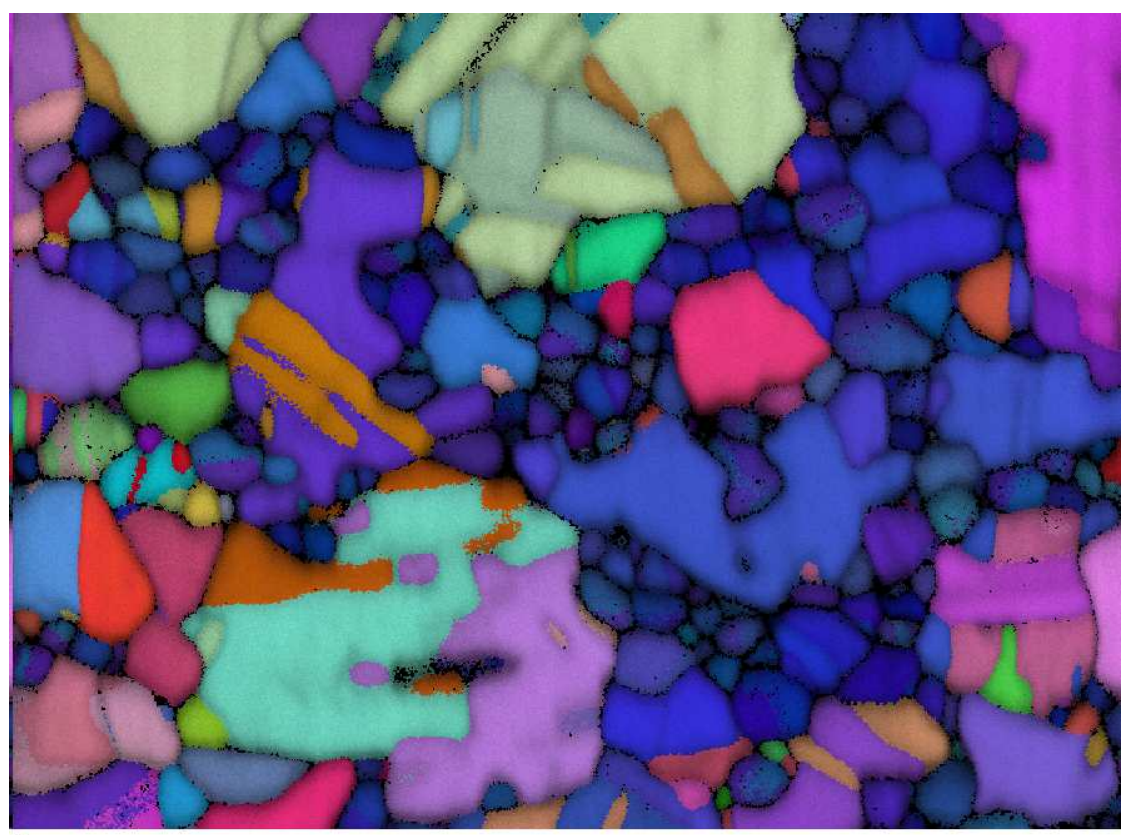

$3 \mu \mathrm{m}$

FIG. 1. Orientation map (relative to the film surface normal) for a partially treated CdTe film using a $10 \mathrm{~nm}$ step size.
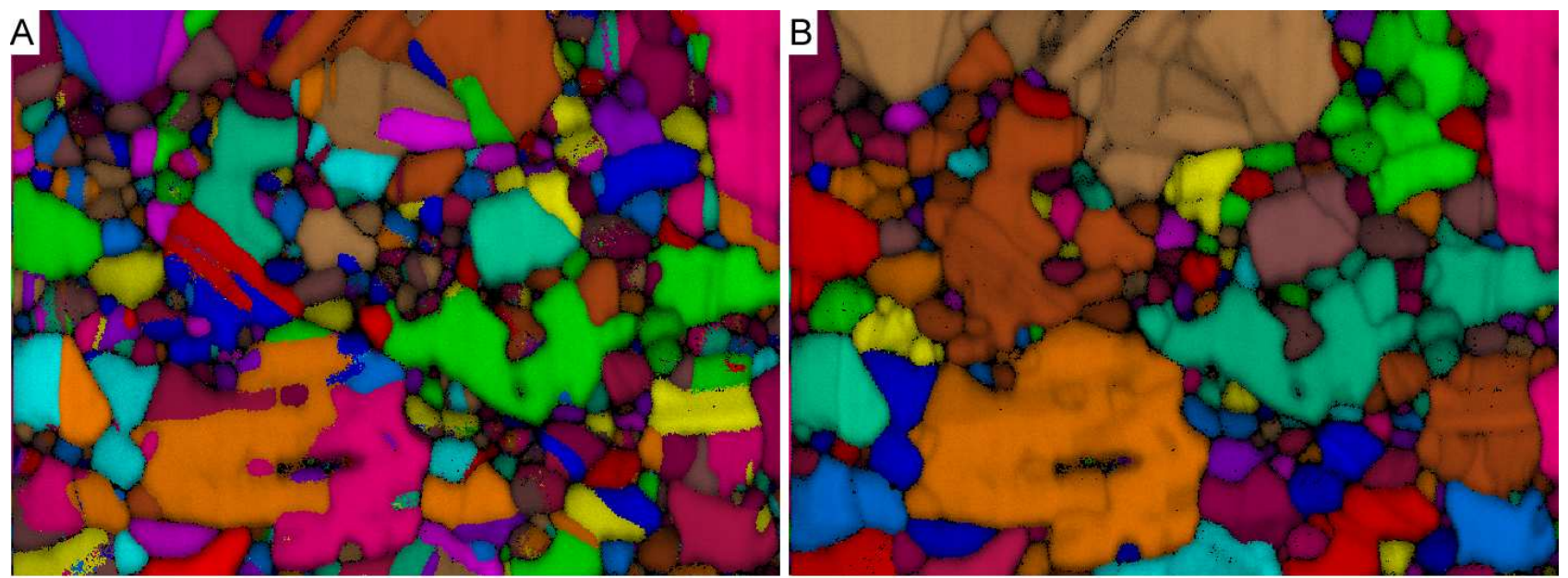

$3 \mu \mathrm{m}$

FIG. 2. Grain maps where grains are randomly colored for grains defined A) including twin boundaries and B) excluding twin boundaries during the point grouping algorithm. 